\title{
Effects of Val34Leu and Val35Leu polymorphism on the enzyme activity of the coagulation factor XIII-A
}

\author{
II-Ha Lee ${ }^{1}$, Soo-II Chung $^{2}$ and Soo-Young \\ Lee $^{1,3}$ \\ ${ }^{1}$ Department of Natural Sciences \\ Chemistry Section, College of Medicine \\ The Catholic University of Korea \\ 505 Banpo-dong, Socho-gu \\ Seoul 137-701, Korea \\ ${ }^{2}$ International Vaccine Institute \\ Kwanak P.O. Box 14, Seoul 151-600, Korea \\ ${ }^{3}$ Corresponding author: Tel, 82-2-590-1263; \\ Fax, 82-2-592-7068; E-mail, Isyng@catholic.ac.kr
}

Accepted 15 November 2002

Abbreviations: PMSF, phenylmethylsulfonyl fluoride; AP, activation peptide; HFXIII-A, human factor XIII A subunit; rFXIII-A, recombinant FXIII-A; RabFXIII-A, rabbit FXIII-A

\begin{abstract}
Change in fibrin stabilizing activity of factor XIII A subunit (FXIII-A) caused by a specific mutation, Val34Leu, is recently implicated to incidences of pathophysiology of thrombosis. In an effort to understand the effect of Val34Leu on enhanced catalytic role of FXIII-A, wild type human factor XIII A (HFXIII-A) and mutant HFXIII-A: HFXIII-A (V34L), HFXIII-A (V35L) and HFXIII-A (V34L/V35L) cDNA were expressed in E.coli system where the purified recombinant FXIII-A (rFXIII-A) showed a similar specific transglutaminase activity comparable to the human native FXIII-A from platelet. Using these rFXIII-A mutants, the activation kinetics by thrombin and the enzymatic properties of the activated rFXIII-A were characterized. rFXIII-A (V34L) and rFXIII-A (V34L/V35L) mutants were activated by thrombin much faster than those of wild type IFXIII-A and V35L variant. However, the activated rFXIII-A and mutants showed the identical catalytic efficiency as measured by in vitro assay. These results suggest that ready activation caused by a specific mutation of neighboring thrombin cleavage site(s) in the activation peptide of FXIII-A like V $34 \mathrm{~L}$ resulted in the real-time amount of the activated factor XIII-A that could influence the outcome of fibrin stabilization in vivo such as $\alpha_{2}-$ plasmin inhibitor crosslinking to fibrin, a reaction
\end{abstract}

known to be dependent on the initial concentration of active factor-XIII-A.

Keywords: Factor XIII, Val34Leu, Val35Leu, catalytic efficiency, site-directed mutagenesis

\section{Introduction}

The cross-linking of fibrin by activated blood coagulation factor XIII (FXIII) is the last enzymatic step in the cascade of blood coagulation. FXIII $A$ subunit (FXIII-A), transglutaminase (EC 2.3.2.13) produced by thrombin activation of the protransglutaminase, catalyzes the covalent intermolecular polymerization of fibrin, through formation of $\varepsilon$-( $\gamma$-glutamyl) lysine crosslinks during blood clotting (Mandel, 1971; Folk and Chung, 1973; Fork and Finlayson, 1973; 1977; Losowsky and Miloszewiski, 1977). A thorough investigation has been made in understanding the molecular and catalytic properties of the FXIII during past 5 decades (Chung et al., 1974; Gorman and Folk, 1980; Ariaens et al., 2002).

The biochemical and physiological effects exerted by a specific mutation, Val34Leu, which is earlier reported to have greater transglutaminase activity (Kangsadalampai and Board, 1998) and associated with incidences with deep vein thrombosis and myocardial infarction are somewhat perplexing although there is reports that the Val34Leu polymorphism affects the function of FXIII by increasing the rate of FXIII activation by thrombin (Ariaens et al., 2000; Wartiovaara et al., 2000). Recently, Lee et al. (2001b) have studied the structure/function relationship of the FXIII-A by comparing enzyme isolated from different animal species. Rabbit factor XIII-A (RabFXIII-A) in comparison with the human enzyme showed significantly greater the kinetic efficiency defined by the specific assay system using methylamine incorporation into acetylated oxidized $B$ chain of insulin $\left(K_{c a t}\right.$ / $K_{M}$ : 68.37 for RabFXIII-A; 20.2 for HFXIII-A). Structural properties (primary, secondary structure and $3 \mathrm{D}$ structure coordinates) of each enzymes from rabbit and human were quite similar except greater variances in amino acid sequences in the activation peptide and found residues near the thrombin cleavage site (Arg37) as Leu34 and Leu35 in rabbit enzyme in place of Val34 and Val35 of human enzyme.

In an effort to clarify the possible influence of Leu34 or Leu35 polymorphism on the enhanced catalytic ef- 
ficiency of FXIII-A, recombinant HFXIII-A (rFXIII-A) mutants were prepared in which Val34 or Val35 was replaced by Leu using site-directed mutagenesis, expressed in E.coli, and the activation profile and enzymatic properties are characterized.

\section{Materials and Methods}

\section{Materials}

Sodium citrate, citric acid, sodium chloride, calcium chloride, thrombin and phenylmethylsulfonyl fluoride (PMSF) were obtained from Sigma Chemical Co. (St. Louis, MO, USA). QuikChange site-directed mutagenesis kit, ECL western blotting reagents, expression vector $\mathrm{pQE} 31$ and $\left[1,4-{ }^{14} \mathrm{C}\right]$ putrescine were purchased from Stratagene (La Jolla, CA, USA), Amersham Pharmacia Biotech (Piscatway, NJ, USA), Qiagen $\mathrm{GmbH}$ (Hilden, Germany) and Perkin-Elmer Inc. (Boston, MA, USA), respectively. HFXIIIA cDNA was kindly provided by Prof. J. M. Jung (Suwon University, Suwon, Korea).

\section{Site-directed mutagenesis}

The 2.1-kb fragment between the Bg/ll and Hindlll restriction sites of the HFXIII-A cDNA generated by PCR using primers containing the restriction sites was subcloned between the BamHI and HindllI restriction sites of pQE31 (Qiagen) to generate plasmids pQE31HFXIII-A. To introduce the V34L, V35L, and V34L/ $\mathrm{V} 35 \mathrm{~L}$ substitutions, the following pairs of oligonucleotide primers were used: P(V34L)5 (5'-GAG CTT CAG GGC CTG GTG CCC CGG GGC-3') and P(V34L)3 (5'-GCC CCG GGG CAG CAG GCC CTG AAG CTC-3'), P(V35L)5 (5'-GAG CTT CAG GGC GTG CTG CCC CGG GGC GT-3') and P(V35L)3 (5'-ACG CCC CGG GGC AGC ACG CCC TGA AGC TC-3'), P(V34L/V35L)5 (5'-GAG CTT CAG GGC CTG CTG CCC CGG GGC GT-3') and P (V34L/V35L)3 (5'-ACG CCC CGG GGC AGC AGG CCC TGA AGC TC-3'). All HFXIII-A mutants, pQE31-HFXIII-A (V34L), pQE31-HFXIII-A (V35L) and pQE31-HFXIII-A (V34L/ V35L), were generated from the PQE31-HFXIII-A plasmid using the QuikChange site-directed mutagenesis kit (Stratagene) following the manufacturer's protocol. Mutations were verified by DNA sequencing.

\section{Expression of FXIII-A in E.coli}

Recombinant wild-type and mutant HFXIII-A (V34L), HFXIII-A (V35L) and HFXIII-A (V34L/V35L) polypeptides were expressed in E.coli strain JM109 containing plasmids pQE31-HFXIII-A, pQE31-HFXIII-A (V34L), pQE31-HFXIII-A (V35L) and PQE31-HFXIII-A (V34L/ $\mathrm{V} 35 \mathrm{~L})$, respectively. Bacteria harboring the FXIII-A ex- pression plasmids were grown in LB medium overnight at $37^{\circ} \mathrm{C}$ from single colony. $100 \mathrm{ml}$ of LB medium containing $50 \mu \mathrm{g} / \mathrm{ml}$ of ampicillin was inoculated with the overnight LB culture $(1: 100)$ and grown at $37^{\circ} \mathrm{C}$ until the cultures reached $A_{590}=0.6$. At this point, the cultures were cooled and grown at $23^{\circ} \mathrm{C}$ for 18 $\mathrm{h}$ in the presence of $0.1 \mathrm{mM}$ IPTG. The bacteria were harvested by centrifugation, washed and resuspended in $3 \mathrm{ml}$ of lysis buffer $(20 \mathrm{mM} \mathrm{CaCl}, 150 \mathrm{mM}$ of $\mathrm{NaCl}, 0.5 \mathrm{mM}$ PMSF, $50 \mathrm{mM}$ Tris, $\mathrm{pH}$ 7.6). The suspension was sonicated three times for $10 \mathrm{~s}$ each with $20 \mathrm{~s}$ between bursts. The lysates were centrifugated at $13000 \mathrm{rpm}$ for $15 \mathrm{~min}$. The supernatants were assayed for FXIII activity. For a large-scale preparation, 5-liter culture was performed and cells harvested were collected by centrifugation. The cell lysate was adsorbed on DE-52 (Whatman, Inc., Clifton, NJ, USA) column $(2 \times 5 \mathrm{~cm})$ equilibrated with $0.05 \mathrm{M}$ Trisacetate, $\mathrm{pH} 6.5$ containing $0.5 \mathrm{mM}$ EDTA and washed with the same equilibration buffer until eluant showed O.D. less than 0.01. Factor XIII-A was eluted with 0.3 $M$ salt in the equilibration buffer and adsorbed in the Casein-linked affinity CL-Sephacryl gel. rFXIII-A was eluted with $0.5 \mathrm{M} \mathrm{NaCl}$ in the equilibration buffer. Eluted enzyme showed close to $90 \%$ purity.

\section{Immunoblotting and immunoquantitation}

The supernatants obtained as above were separated on a $12 \%$ SDS-polyacrylamide gel. After electrophoresis, the gel was transferred to a nitrocellulose membrane in an XCell ${ }^{\mathrm{TM}}$ blot module (Novex, San Diego, CA, USA). The membrane was incubated for $1 \mathrm{~h}$ with antibody. After washing the blots to remove unbound antibodies, the proteins of interest were visualized with the ECL system (Amersham, Buckinghamshire, UK). Immunoquantitation was carried out by densitometer.

\section{Factor XIII activity assay}

The thrombin activated FXIII-A activity was measured by an incorporation of $\left[1,4-{ }^{14} \mathrm{C}\right]$ putrescine $(100 \mu \mathrm{Ci}$ / $\mu$ mole) into the succinylated Hammersten-casein assay as described. The assay solution contained 0.1 $\mathrm{M}$ Tris-acetate, $\mathrm{pH}$ 8.0, $1 \mathrm{mM}$ EDTA, $10 \mathrm{mM} \mathrm{CaCl}_{2}$, $5 \mathrm{mM}$ DTT, $0.5 \%$ Lubrol (Sigma) and $1 \%$ succinylated Hammersten-casein. The reaction was started with an addition of $10 \mu \mathrm{l}$ of $0.5 \mu \mathrm{Ci}$ radioactive putrescine into the $0.45 \mathrm{ml}$ of assay mixture containing FXIII-A and thrombin at $37^{\circ} \mathrm{C}$ and stopped by addition of $4 \mathrm{ml}$ $10 \%$ cold TCA solution. The casein precipitate was filtered on the glass filter (Whatman GF/A) and the radioactivity was measured by scintillation counter (Beckman Inc., Fullerton, CA, USA).

The rate studies were carried out in $0.1 \mathrm{mM}$ Tris- 
acetate, pH 7.5 containing $30 \mathrm{mM} \mathrm{CaCl}, 0.5 \mathrm{mM}$ EDTA, $10 \mathrm{nM}$ DTT, varying concentration of succinylated Hammersten-casein and $5 \mu \mathrm{M}\left[1,4-{ }^{14} \mathrm{C}\right] \mathrm{pu}-$ trescine (specific activity of $100 \mu \mathrm{Ci} / \mu$ mole) and with appropriate amount of enzyme at $25^{\circ} \mathrm{C}$. The incubation time $(20 \mathrm{~min})$ was selected from the linear rate of putrescine incorporation where the total amount of amine incorporated is less than $10 \%$ of the total amount of putrescine concentration. The data for initial velocity of putrescine incorporation into casein substrate were found to conform to a modified double displacement mechanism (Folk and Chung, 1973)

\section{$v=V_{a b} A B / K_{a h} K_{a t}+K_{a h} B+K_{b t} A+A B$}

For clarity, the definitions of certain kinetic constants for this mechanism are given earlier (Folk and Chung, 1973). The kinetic constant subscript $h$ designates the Michaelis constant for hydrolysis and $t$ the Michaelis constant for transfer. A designates the peptide-bound glutamine substrate (succinylated Hammersten-Casein) concentration and $B$, the primary amine substrate (putrescine) concentration. All curve fitting was performed by means of MLAB, an interactive nonlinear least square curve fitting system.

\section{Clot lysis assay}

Human fibrinogen was prepared by a modification of the method of Mosesson et al. (1966). Purified fibrinogen was dialyzed against $3 \mathrm{mM}$ of urea and 5 $\mathrm{mM}$ of $\mathrm{CaCl}_{2}$ to inactivate contaminated FXIII. The clottability of the fibrinogen was higher than 95\% when thrombin was added. Fibrin clot plate was prepared from FXIIl-free fibrinogen ( $3 \mathrm{mg} / \mathrm{ml}$ ) by adding 1 unit of thrombin and the lysates of rFXIIIA

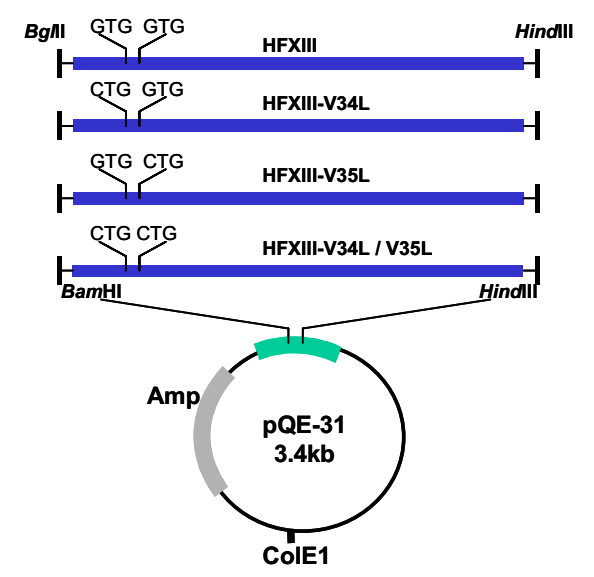

Figure 1. Schematic diagram of the genetically engineered FXIII-A cDNA and expression vector. The detailed procedures for the construction of FXIII-A for expression in E.coli are described under Materials and Methods. The black bar indicates the protein coding region. The GTG and GTG stand for Val34 and Val35 in wild type HFXIII-A and CTG for Leu. wild type, rFXIIIA (V34L), rFXIIIA (V35L) and rFXIIIA (V34L/V35L), respectively at $37^{\circ} \mathrm{C} .1 \mu \mathrm{g}$ of plasmin was overloaded on fibrin clot plate. After $1 \mathrm{~h}$ incubation at $37^{\circ} \mathrm{C}$, the diameter of the clear zone was measured.

\section{Results}

\section{Expression and immunoblotting of recombinant HFXIIIA wild type and mutants}

A Leu34 polymorphism in HFXIII-A was reported to show greater transglutaminase activity than wild type Val34 (Kangslalampai and Board, 1998). Also RabFXIII-A whose activation peptide having Leu34 and Leu35 residues possessed enhanced specific transglutaminase activity in comparison with the human enzyme having Val34 and Val35 residues. To delineate a possible association of Leu34 and Leu35 polymorphism with the factor XIII-A catalytic activity, mutants-rFXIII-A (V34L), rFXIII-A (V35L) and rFXIII-A (V34L/V35L) were constructed (Figure 1) and expressed in E.coli. Figure 2 shows comparable expression levels of respective factor XIII-A mutants. In order to confirm the rFXIII-A expressed in E.coli retain their intrinsic catalytic properties, transglutaminase activities of the FXIII-A and mutants were compared with factor XIII-A from platelet in vitro amine incorporation assay as described in the method. The specific activity of the thrombin-activated purified rFXIII-A (wild type) was similar to that of purified human FXIII from platelets (Table 1). For the rest of

Table 1. Relative activity of purified and recombinant FXIII.

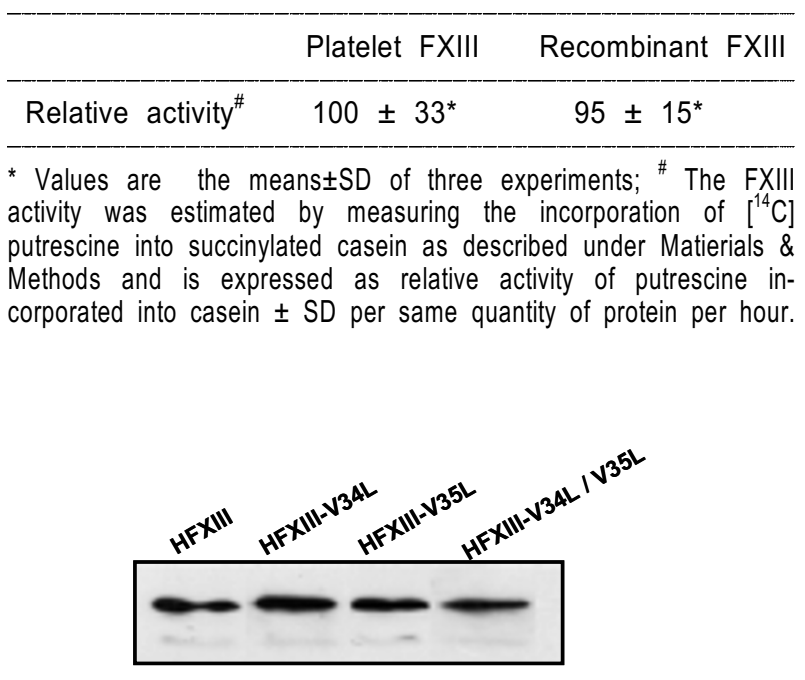

Figure 2. Expression of wild type and mutants of $\mathrm{rFXIII-A}$ in E.coli. The soluble fraction of total lysate from bacteria was electrophoresed on a $12 \%$ SDS-PAGE, blotted and probed with anti-HFXIII-A antibody. 


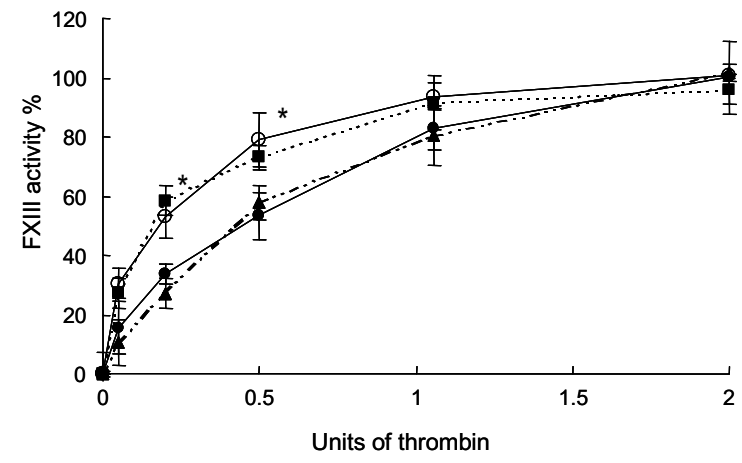

Figure 3. FXIII activity of quantified soluble fraction prepared from E.coli. FXIII activity was measured by $\left[1,4-{ }^{14} \mathrm{C}\right]$ putrescine incorporation assay with various concentrations of thrombin for $30 \mathrm{~min}$.

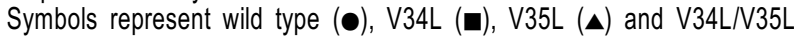
(○) mutants, respectively. Statistical analyses were carried out using Students $t$ test. ${ }^{*} P<0.05$.

assay we have simplified the assay method by using the cell lysate directly since the $E$. coli does not contain any transglutaminase activity and the amount of rFXIII-A present was determined by immuno-assay as stated in the method.

\section{FXIII activation by thrombin}

In previous reports, mutant $\gamma$ containing Leu34 exhibited 1.5 or 2-fold higher FXIII activity than the wild type rFXIII-A Val34 at insufficient thrombin activation (Muszbek et al., 2000). In order to examine the efficiencies of enzymatic activation of wild type rFXIIIA, rFXIII-A (V34L), rFXIII-A (V35L) and rFXIII-A (V34L/ $\mathrm{V} 35 \mathrm{~L})$ polypeptide by various concentration of thrombin, the FXIII activity of rFXIII variants were investigated under $0,0.05,0.2,0.5,1$ and 2 unit of thrombin for $30 \mathrm{~min}$ at $37^{\circ} \mathrm{C}$. rFXIII-A (V34L) and rFXIII-A (V34L/V35L) showed 2-fold higher FXIII activity than rFXIII-A and rFXIII-A (V35L) by 0.2 unit of thrombin and there was no difference of FXIII activity between rFXIII-A (V34L) and rFXIII-A (V34L/ V35L). The V35L mutant showed no enhanced activation. In addition, FXIII activities of rFXIII-A wild type and mutants with 2 unit of thrombin were nearly identical (Figure 3). The kinetic study of rFXIII-A (V34L) and activation with 0.02 units of thrombin where the thrombin was inactivated with $2 \mathrm{mM}$ PMSF at each time point, showed a clear difference on the rate of thrombin hydrolysis of mutant with wild type (data not shown).

\section{Catalytic efficiency}

In order to confirm whether variance in amino acid sequence in the activation peptide effects possible catalytic properties of transglutaminase activity of
Table 2. Catalytic efficiency analysis of HFXIII-A wild type and mutants.*

\begin{tabular}{llll}
\hline Mutation & $K_{M}(\mathrm{M})$ & $K_{\text {cat }}(\mathrm{s}-1)$ & $\begin{array}{c}K_{\text {cat }} / K_{M} \\
\left(\mathrm{M}^{-1} \mathrm{~s}^{-1}\right)\end{array}$ \\
\hline Wild type & $2.5 \times 10^{-5}$ & $6.9 \times 10^{-1}$ & $2.7 \times 10^{2}$ \\
V34L & $3.2 \times 10^{-5}$ & $9.2 \times 10^{-1}$ & $2.8 \times 10^{2}$ \\
V35L & $2.8 \times 10^{-5}$ & $6.5 \times 10^{-1}$ & $2.3 \times 10^{2}$ \\
V34L/V35L & $3.1 \times 10^{-5}$ & $8.4 \times 10^{-1}$ & $2.7 \times 10^{2}$ \\
\hline
\end{tabular}

*Recombinant wild type and mutants of FXIII-A were incubated with succinylated Hammersten-casein contatining radioactive putrescine and thrombin. The enzyme kinetic constants were calculated as described in Materials and Methods.

rFXIII-A mutant, we investigated that catalytic efficiencies of the different forms of rFXIII-A following full activation of rFXIII-A with 2 units of thrombin as described in the methods. For the rFXIII-A (V34L) and rFXIII-A (V34L/V35L), the $K_{M}$ value and $K_{c a t}$ did not show any significant differences (Table 2). In addition, there were no differences between $\mathrm{rFXIII-A}$ wild type and rFXIII-A (V35L) in the $K_{M}$ and $K_{c a t}$ value. The resultant catalytic efficiency $\left(K_{c a t} / K_{M}\right.$ value $)$ of the rFXIII-A (V34L) and rFXIII-A (V34L/V35L) was not increased compared with that of the rFXIII-A wild type and rFXIII (V35L). Within the experimental error, the kinetic efficiency of the wild type and mutants rFXIII-A were almost identical.

\section{Clot lysis}

Factor XIII catalyzed crosslinking of fibrin chains to form $\gamma$-dimer and $\alpha$-polymer and also crosslinking of $\alpha_{2}$-plasmin inhibitor to fibrin $\alpha$ chain resulting in the stabilized fibrin polymers. Thus, the degree of crosslinking should dependent on the amount of activated FXIII-A and in turn will dependent on the level of thrombin that also catalyzes the fibrin gel formation. In addition, the crosslinking of $\alpha_{2}$-plasmin inhibitor to fibrin occurs during the limited time frame of fibrin polymerization. The clot lysis assay involves the number of factors that are interdependent to each other. Here, we have examined the effect of rFXIII-A mutants with the fibrinogen devoid of plasma FXIII and limited levels of thrombin to effect the fibrin polymer formation as well as rFXIII activation to evaluate whether there rFXIII-A wild type and rFXIII-A mutants affect cross-linking of fibrin chain. The fibrin gel plate was employed for this assay. As the plasmin is allowed to diffuse from the center well, the opaqueness of fibrin plate to become clear.

The size of clear zone formed by lysis should reflect the degree of fibrin stabilization. The control fibrin without factor XIII-A showed greater width of 


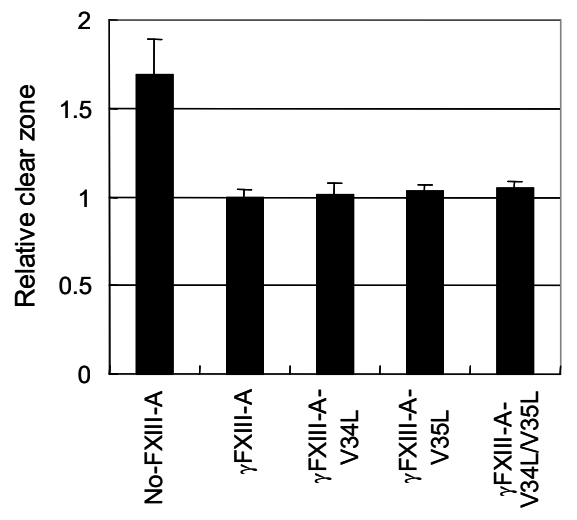

Figure 4. Clot lysis assay. Fibrin clot was prepared from FXIII-free fibrinogen adding 1 unit of thrombin and the soluble fraction of total lysate from wild type, V34L, V35L and V34L/V35L rFXIII-A. The clear zone by plasmin was measured.

zone area. However, the difference among the different forms of rFXIII-A could not be found (Figure 4). Again, this kind of diffusion assay which requires extended time period showed no distinction among the different variants of rFXIII-As.

\section{Discussion}

HFXIII-A subunit is synthesized as a monomeric form and exists in a dimeric zymogen where the 37 $\mathrm{N}$-terminal amino acid activation peptide (AP) of each subunit crosses over dimer interface and partially occludes the opening of the catalytic cavity in the second subunit and preclude substrate binding to the zymogen (Arg-11 of first monomer binds to Asp-343 of core domain in second monomer) (Sadasivan and Yee, 2000).

The natural polymorphoism of factor XIII A chain where the amino acid substitution at position Val34 with leucine does influences the activity of FXIII-A in vivo where both a milieu of substrates for factor XIII and efficient control system of thrombin activity exist. To investigate how a single substitution, Val34 to Leu in FXIII-A that are situated on periphery away from the active site cleft could induce alteration of catalytic property of enzyme, we have prepared rFXIII-A variants with substitution at Val34Leu, Val35Leu and Val34Leu/Val35Leu and examined the efficiencies of enzymatic activation by thrombin and catalytic efficiency of the activated enzyme using two different substrates; one with fibrinogen and the other with non-fibrinogen. A single substitution at a position V34L allowed 2-fold higher FXIII activity than rFXIII-A and rFXIII-A (V35L) in the presence of low concentration of thrombin. This difference was diminished with either extending incubation time with thrombin or at elevated thrombin concentration suggesting that substrate efficiency for thrombin was enhanced by P4 subsite substitution.

There were no significant differences in the $K_{M}, K_{c a t}$ and $K_{c a t} / K_{M}$ values examined with non-fibrinogen substrate (casein) among rFXIII-A wild type and mutants. A similar results were obtained with fibrinogen substrate with various FXIII-A mutants via plasmin mediated assay for stabilized fibrin as a degree of FXIII-A catalyzed fibrin crosslinking.

Ariaens et al. (2000) and Wartiovaara et al. (2000) have reported that HFXIII-A V34L polymorphism accelerates activation of FXIII by thrombin. We also have reported that the amino-terminal AP domain of RabFXIII-A which is quite different from human, especially Leu34 and Leu35 (Lee et al., 2001b), was far more sensitive to thrombin in comparison with human plasma XIII independent of the enhanced catalytic efficiency (data not shown). In light of these reports, rFXIII-A (V34L) and rFXIII-A (V34L/V35L) appears to be activated more rapidly by thrombin, and no apparent enhancement of catalytic efficiency measured in a strictly defined assay system. The enhanced effect of rFXIII-A (V34L) and rFXIII-A (V34L/ $\mathrm{V} 35 \mathrm{~L}$ ) without effect of rFXIII-A (V35L) on the thrombin activation and consequencial increment of factor XIII catalysis under a limited thrombin levels in the present study supports view that the Val34Leu polymorphism in the A subunit affects factor XIII catalyzed reactions in physiological system. Futhermore, this study demonstrated the definite influence of Val34Leu in the RabFXIII-A which has Val34Leu and Val35Leu, in the enhanced catalytic role in vivo. But, it is interesting to note that one closer residue Val35Leu does not effect the thrombin catalytic efficiency.

The rate of vascular fibrinolysis is not only dependent on the concentration of the interacting components of the fibrinolytic enzyme system, i.e., plasminogen, plasminogen activator, $\alpha_{2}$-plasmin inhibitor, plasminogen activator inhibitor and plasminogen activator receptor on the vascular endothelium, but is also regulated by the extent of fibrin cross- linking with $\alpha_{2}$-plasmin inhibitor where the reaction is solely dependent on the initial concentration of FXIII-A (Chung et al., 1996; Francis and Marder, 1988). In the present study, no significant differences in the purified fibrin clot lysis among the different forms of rFXIII-As could be found. Perhaps, test should be on the real time assay on fibrin stabilization including $\alpha_{2}$-plasmin inhibitor.

\section{References}

Ariaens RA, Lai TS, Weisel JW, Greenberg CS, Grant PJ. 
Role of factor XIII in fibrin clot formation and effects of genetic polymorphisms. Blood 2002;100:743-54

Arins RA, Philippou H, Nagaswami C, Weisel JW, Lane DA, Grant PJ. The factor XIII V34L polymorphism accelerates thrombin activation of factor XIII and affects cross-linked fibrin structure. Blood 2000;96:988-95

Chung SI, Lee SY, Uchino R, Carmassi F. Factors that control extravascular fibrinolysis. Semin Thromb Hemost 1996;22:479-88

Chung SI, Lewis MS, Folk JE. Relationships of the catalytic properties of human plasma and platelet transglutaminases (activated blood coagulation factor XIII) to their subunit structures. J Biol Chem 1974;249:940-50

Folk JE, Chung SI. Molecular and catalytic properties of transglutaminases. Adv Enzymol Relat Areas Mol Biol 1973; 38:109-91

Folk JE, Finlayson JS. The epsilon-(gamma-glutamyl) lysine crosslink and the catalytic role of transglutaminases. Adv Protein Chem 1977;31:1-133

Francis CW, Marder VJ. Increased resistance to plasmic degradation of fibrin with highly crosslinked alpha-polymer chains formed at high factor XIII concentrations. Blood 1988; 71:1361-65

Gorman JJ, Folk JE. Structural features of glutamine substrates for human plasma factor XIIla (activated blood coagulation factor XIII). J Biol Chem 1980;255:419-27

Kangsadalampai S, Board PG. The Val34Leu polymorphism in the A subunit of coagulation factor XIII contributes to the large normal range in activity and demonstrates that the activation peptide plays a role in catalytic activity. Blood 1998;92:2766-70

Lee SY, Chang SK, Lee IH, Kim YM, Chung SI. Depletion of plasma factor XIII prevents disseminated intravascular coagulation-induced organ damage. Thromb Haemost 2001a; 85:464-9

Lee SY, Lee IH, Oh JT, Chung SI. Characterization of enhanced catalytic efficiency of rabbit Factor XIII in comparison with human enzyme. 2001b; XVIII International Society of Thrombosis and Haemostasis, Paris, France

Losowsky MS, Miloszewski KJ. Factor XIII. Br J Haematol 1977;37:1-5

Mandel EE. The fibrin-stabilizing factor. A decade of progress. Ann Clin Lab Sci 1971;1:92-9

Mosesson MW, Sherry S. The preparation and properties of human fibrinogen of relatively high solubility. Biochemistry 1966; 5:2829-35

Muszbek L. Deficiency causing mutations and common polymorphisms in the factor XIII-A gene. Thromb Haemost 2000; 84:524-7

Sadasivan C, Yee VC. Interaction of the factor XIII activation peptide with alpha-thrombin. Crystal structure of its enzymesubstrate analog complex. J Biol Chem 2000;275:36942-8

Wartiovaara U, Mikkola H, Szoke G, Haramura G, Karpati L, Balogh I, Lassila R, Muszbek L, Palotie A. Effect of Val34Leu polymorphism on the activation of the coagulation factor XIII-A. Thromb Haemost 2000;84:595-600 